\title{
Pig lung immune cytokine response to the swine influenza virus and the Actinobacillus pleuropneumoniae infection
}

\author{
Ewelina Czyżewska-Dors, Arkadiusz Dors, Krzysztof Kwit, \\ Ewelina Stasiak, Małgorzata Pomorska-Mól \\ Department of Swine Diseases, \\ National Veterinary Research Institute, 24-100 Pulawy, Poland \\ mpomorska@piwet.pulawy.pl
}

Received: May 8, 2017

Accepted: August 31, 2017

\begin{abstract}
Introduction: The aim of this study was to evaluate and compare the local innate immune response to the swine influenza virus (SIV) and Actinobacillus pleuropneumoniae (App) infection in pigs. Material and Methods: The study was performed on 37 seven-week-old pigs, divided into four groups: App-infected $(\mathrm{n}=11)$, App+SIV-infected $(\mathrm{n}=11), \mathrm{SIV}$-infected $(\mathrm{n}=11)$, and control $(n=4)$. Lung samples were collected, following euthanasia, on the $2^{\text {nd }}$ and $4^{\text {th }}$ dpi (three piglets per inoculated group) and on the $10^{\text {th }}$ dpi (remaining inoculated and control pigs). Lung concentrations of IL-1 $\beta$, IL-6, IL-8, TNF- $\alpha$, IL-10, IFN- $\alpha$, and IFN- $\gamma$ were analysed with the use of commercial porcine cytokine ELISA kits. Results: Lung concentrations of IL-1 $\beta$, IL-6, IL-8, TNF- $\alpha$, IFN- $\alpha$, and IFN- $\gamma$ were induced in SIV-infected and App+SIV-infected pigs. In the lung tissue of App-infected pigs, only concentrations of IL-1 $\beta$, IL-6, IL-8, and IFN- $\gamma$ were elevated. Additionally, in App+SIV-infected pigs, significantly greater concentrations of IL-1 $\beta$, IL-8, and IFN- $\alpha$ were found when compared with pigs infected with either SIV or App alone. In each tested group, the lung concentration of IL-10 remained unchanged during the entire study. Conclusion: The results of the study indicate that the experimental infection of pigs with SIV or App alone and co-infection with both pathogens induced a local lung inflammatory response. However, the local cytokine response was considerably higher in co-infected pigs compared to singleinfected pigs.
\end{abstract}

Keywords: pigs, swine influenza virus, Actinobacillus pleuropneumoniae, local immunity, cytokines.

\section{Introduction}

Porcine respiratory disease complex (PRDC) is a serious health problem in pork production worldwide (19). PRDC can be caused by a combination of viral and/or bacterial agents. The most common pathogens involved in the disease are swine influenza virus (SIV), porcine reproductive and respiratory syndrome virus (PRRSV), Actinobacillus pleuropneumoniae (App), Pasteurella multocida (Pm), and Mycoplasma hyopneumoniae (Mhp) (22). It has been shown that bacterial-viral co-infections can exacerbate the pathogenicity of respiratory diseases $(17,23,26,28)$. SIV, an aetiological agent of swine influenza (SI), is considered a primary pathogen, able to cause the disease in single-infected animals, but it can also act simultaneously with other agents. In an uncomplicated
SIV infection mortality is low $(<1 \%)$ and recovery occurs within 5-7 days (20). However, secondary bacterial infections can increase SI severity and mortality rates $(20,29)$. App, an aetiological agent of fibrino-haemorrhagic necrotising pleuropneumonia, is considered both a primary and secondary pathogen $(8$, 14). At present, there are 16 recognised serotypes of App with a considerable variation in virulence potential (7, 14). In Eastern Europe, the main serotypes are 2, 1, 9, 6, 7 , and 8 , with the dominance of serotype 2 in Poland (15). Respiratory signs due to SI are the result of direct tissue damage by a replicating virus and are also related to the proinflammatory response of the host $(4,24,25$, 30). Additionally, the clinical outcome induced by App is a result of a direct, devastating effect of App exacerbated by the induction of proinflammatory cytokines $(1,6,9,10)$. The aim of the present study was 
to investigate and compare the innate immune response in the lungs of pigs after single or dual inoculation with SIV and App.

\section{Material and Methods}

Virus and bacteria. The recently circulating in Poland avian-like H1N1 SIV (A/Poland/Swine/14131/ 2014; hereinafter referred to as SwH1N1), isolated from the lungs of a pig suffering from acute SI, was used for the inoculation of pigs. App serotype 2 (App2), strain 4226, kindly provided by Marcelo Gottschalk (Université de Montréal, Canada) was used as a bacterial pathogen.

Experimental design. A total of 37 seven-weekold pigs from an influenza and App negative farm were used in the experiment. Animals were randomly divided into four groups: App $(n=11), \operatorname{App}+\operatorname{SIV}(n=11)$, SIV $(n=11)$, and control $(n=4)$. No evidence of streptococcosis or progressive atrophic rhinitis (PAR) was recorded in the herd based on clinical, serological, and pathological examinations.

Prior to the start of the experiment, all pigs were free of the influenza A virus and App2 antibodies, as determined by haemagglutination inhibition assays, using an A/Poland/Swine/14131/2014 (H1N1), A/swine/England/96 (H1N2), A/swine/Flanders/1/98 (H3N2), pdm-like H1N1 (A/swine/Poland/031951/12), and ID Screen APP 2 Indirect (ID.vet) ELISA. They were also free of antibodies to ApxIV, the toxin common to all App serotypes, as determined by an APP-ApxIV Ab Test (IDEXX, USA).

Pigs were housed at a BSL3 animal facility, an independent unit being provided for each group. On day 0 , pigs from SIV and App+SIV groups were inoculated intranasally (IN) with SwH1N1 (10 7 TCID50 in $3 \mathrm{~mL}$ of phosphate-buffered saline (PBS)). Pigs from App and App+SIV groups were challenged IN with App2 $\left(3.9 \times 10^{8} \mathrm{cfu}\right.$ App2 in $3 \mathrm{~mL}$ of PBS). Four mockinoculated pigs with PBS served as controls.

Clinical examination of pigs (rectal temperature, respiratory and general signs) was performed daily, from day 7 before inoculation until 10 days post inoculation (dpi) or until euthanasia (at 2 or 4 dpi). Fever was recorded when the rectal temperature was equal or higher than $40^{\circ} \mathrm{C}$. Three piglets per inoculated group were euthanised at 2 and $4 \mathrm{dpi}$. The remaining inoculated and control pigs were euthanised at $10 \mathrm{dpi}$. Complete necropsy was performed on each animal, with special emphasis on the respiratory tract. Lung lesions (SI-like) were scored, using the method developed by Madec and Kobisch (21) according to the procedure described previously (25). In addition, App-like lesions in the lungs were assessed at necropsy according to the method described previously (27). Samples from cranial, middle, and accessory lobes of the right lung (all lobes separately) were collected during necropsy for estimation of cytokine concentration.
Cytokine concentration in the lungs. The samples from cranial, middle, and accessory right lobes were collected from control and infected pigs (25). One gram of lung tissue was suspended in $1 \mathrm{~mL}$ of PBS, $\mathrm{pH} 7.4$, $(1: 1 \mathrm{w} / \mathrm{v})$ and frozen, before being homogenised. Next, the samples were centrifuged at 2,440 $\times \mathrm{g}$ for $10 \mathrm{~min}$. The supernatants were collected and stored at $-70^{\circ} \mathrm{C}$, for up to a maximum of one month. Cytokine concentration was analysed with the use of porcine cytokine ELISA kits: Porcine IL-8, IL-10, IFN- $\gamma$, and TNF- $\alpha$ from the Invitrogen Corporation (Camarillo, USA); Porcine IL-1 $\beta$ and IFN- $\alpha$ from RayBiotech (Norcross, USA) and IL-6 Pig ELISA Kit from Abcam (Cambridge, UK). Detection limits for the kits were: $6 \mathrm{pg} / \mathrm{mL}$ (IL-1 $\beta$ ), $45 \mathrm{pg} / \mathrm{mL}$ (IL-6), $10 \mathrm{pg} / \mathrm{mL}$ (IL-8), $3 \mathrm{pg} / \mathrm{mL}$ (IL-10), $2 \mathrm{pg} / \mathrm{mL}($ IFN- $\alpha), 2 \mathrm{pg} / \mathrm{mL}($ IFN- $\gamma$ ), and $3 \mathrm{pg} / \mathrm{mL}$ (TNF- $\alpha$ ), respectively. All tests were conducted according to manufacturers' recommendations. The quantity of cytokines was calculated, based on a standard curve for each cytokine, with the use of FindGraph software. For statistical analyses, levels lower than the detection limits were set as detection limit (in $\mathrm{pg} / \mathrm{mL}$ ) minus 1 ( $1 \mathrm{pg} / \mathrm{mL}$ for IFN- $\gamma, 9 \mathrm{pg} / \mathrm{mL}$ for IL-8, and $2 \mathrm{pg} / \mathrm{mL}$ for IL-10).

Statistical analysis. The obtained data were subjected to a W. Shapiro-Wilk test for normality and Levene's test for equality of variances. After rejection of normality and variance homogeneity, differences between means were tested for statistical significance, using a nonparametric Kruskal-Wallis test, with post-hoc multiple comparisons for the comparison of all pairs. Differences were considered as significant at $\mathrm{P}<0.05$. All calculations were performed with Statistica 8.0 (Statsoft).

\section{Results}

Seven piglets inoculated with SwH1N1 and nine inoculated with App had a short-term fever (40.0 $41.0^{\circ} \mathrm{C}$ ). In the group inoculated with both pathogens, long-term (over three days) fever was observed in all animals. The most severe course of the disease was observed in co-infected piglets (all piglets demonstrated at least one clinical sign). In pigs inoculated with SIV only, clinical signs were recorded in 8/11 animals, while in the group inoculated with App, only 5/11 pigs had clinical signs of respiratory infection. No symptoms were observed in control pigs.

All animals from the SIV and App+SIV groups had lung lesions characteristic of SIV infection (lung score from 1 to 9 in SIV and from 1 to 15 in App+SIV). The influenza-like lesions were also observed in 3/11 pigs from the App group (lung score of 1, 1, and 4). The App-like lesions were observed only in animals inoculated with App (App and App+SIV groups). The control pigs showed no pathological lesions. The typical lesions observed in experimental pigs are presented in Fig. 1. 

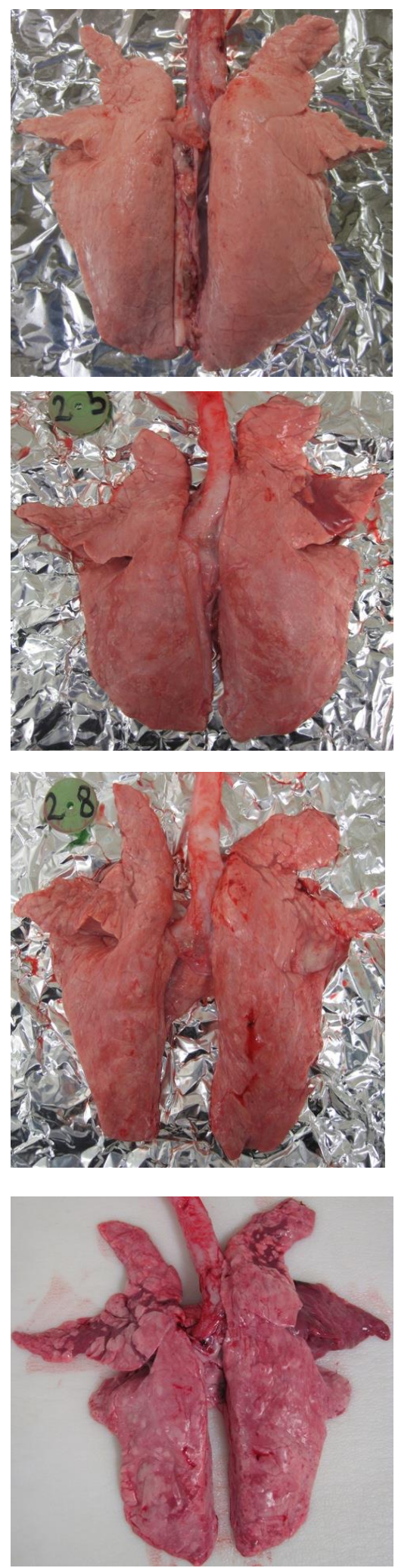
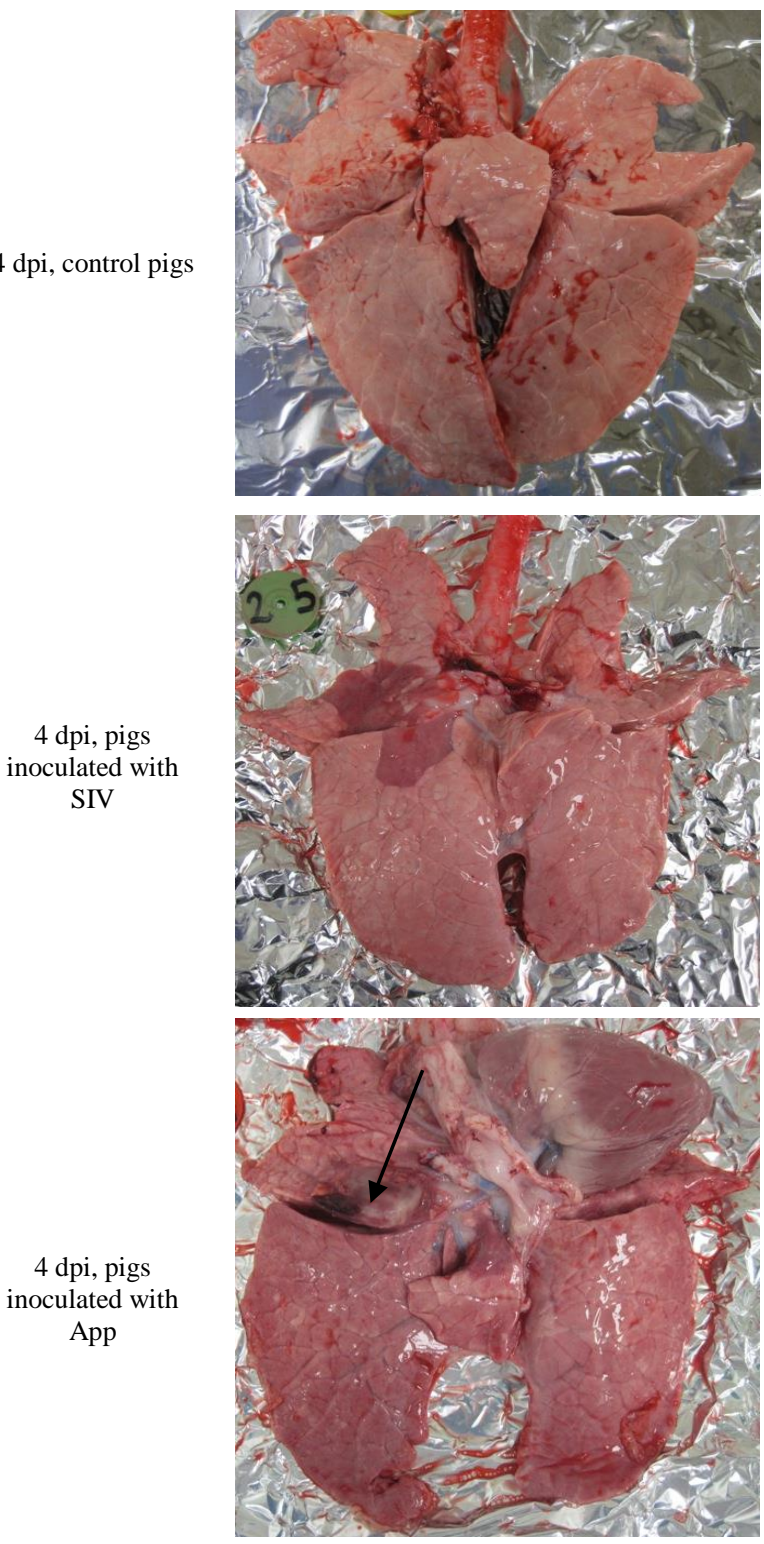

4 dpi, pigs inoculated with App

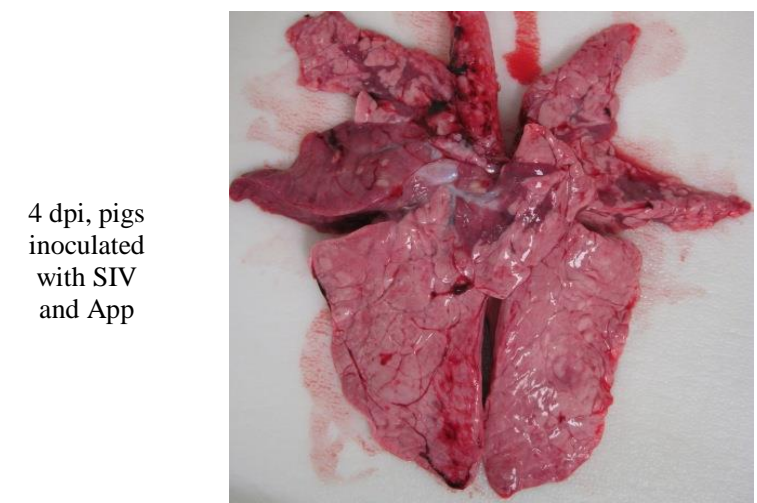

Fig. 1. Gross lung lesions observed on the $4^{\text {th }}$ day post-inoculation (dpi) in pigs singly or doubly inoculated with SIV and/or App

Detailed data on the mean $( \pm \mathrm{SD})$ cytokine concentrations in the lungs of experimental pigs are presented in Fig. 2. In general, lung concentrations of IL-1 $\beta$, IL-6, IL-8, TNF- $\alpha$, IFN- $\alpha$, and IFN- $\gamma$ were induced in the SIV- and App+SIV-infected pigs, while in App-infected pigs, only concentrations of IL-1 $\beta$, IL-6, IL-8, and IFN- $\gamma$ were elevated (Fig. 2). The lung concentration of IL-10 remained unchanged during the entire study in each tested group $(\mathrm{P} \geq 0.05)$. 
On $2 \mathrm{dpi}$, the concentrations of IL-8, IFN- $\gamma$, and IL-6 were significantly higher in all infected groups, compared to controls $(\mathrm{P}<0.05)$ with respect to all evaluated parts of the lung for IL-8, the middle and accessory lobes for IFN- $\gamma$, and the middle lobe for IL$6(\mathrm{P}<0.05)$. In the SIV- and App+SIV-infected groups, a higher concentration of IL-6 in the apical lobes was also noted in comparison to control group. In the App- and App+SIV-infected pigs, significantly higher concentration of IL-1 $\beta$ as compared to control group was observed in all lobes and in apical and middle lobes, respectively. The concentrations of TNF- $\alpha$ and IFN- $\alpha$ were significantly higher in the
SIV- and App+SIV-infected pigs, compared to controls. Furthermore, statistically significant differences in cytokine concentration between the inoculated groups were also detected. In the App+SIV-infected pigs, the concentration of IL- 8 in the middle lobe was significantly greater than in the single-infected groups $(\mathrm{P}<0.05)$. Moreover, the level of IL-8 was significantly higher in the lungs of Appinfected pigs than the SIV-infected group $(\mathrm{P}<0.05)$. At the same time, by contrast, the concentrations of IL-6 and IFN- $\alpha$ were significantly greater in the SIVinfected group than in the App-infected group $(\mathrm{P}<0.05)$.
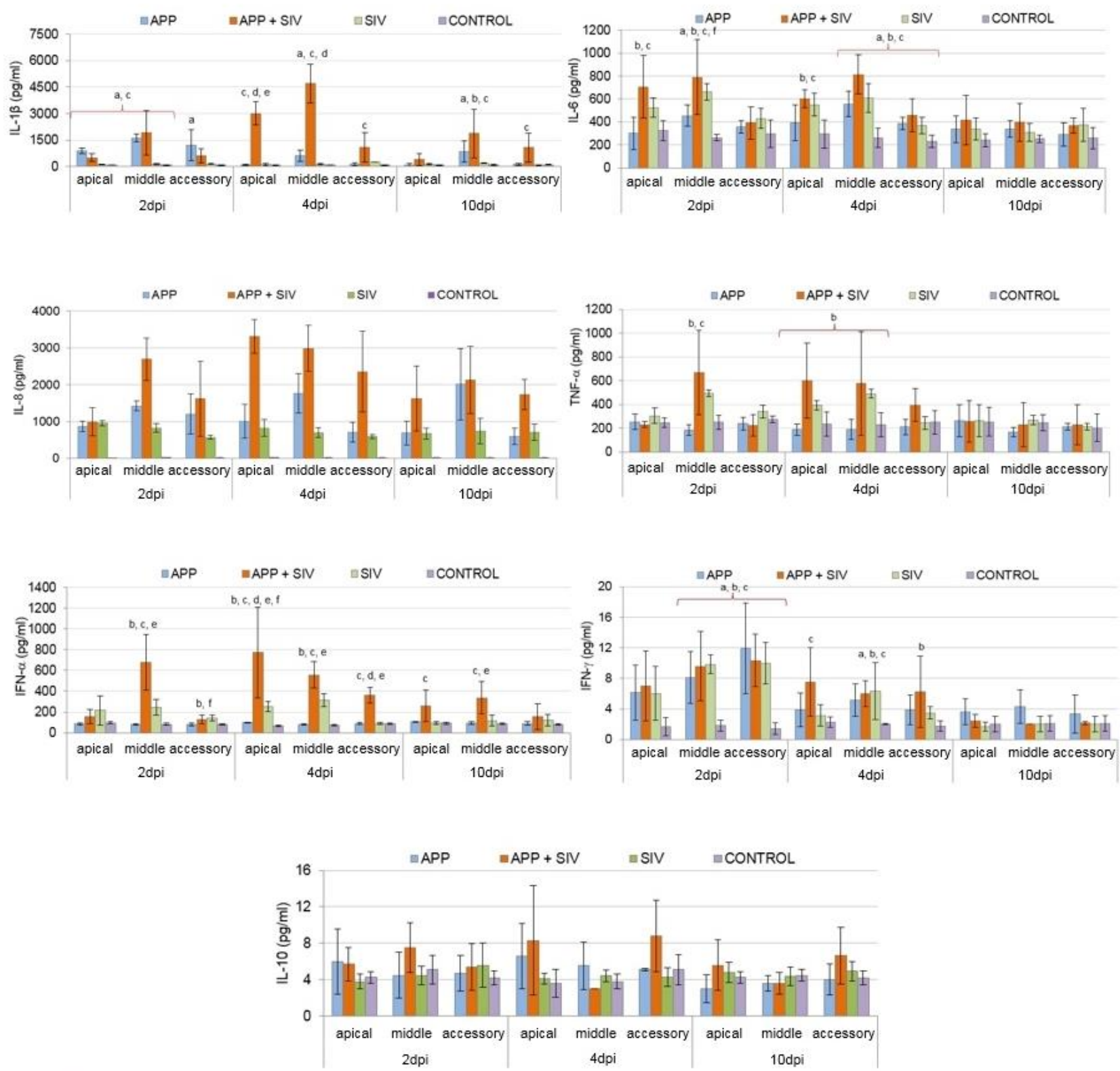

Fig. 2. Concentration of cytokines in lung samples from pigs inoculated with SIV and/or App; a - significant difference between App-infected group and control group; $\mathrm{b}$ - significant difference between SIV-infected group and control group; $\mathrm{c}$ - significant difference between App+SIVinfected group and control group; $\mathrm{d}$ - significant difference between App+SIV-infected group and App-infected group; $\mathrm{e}$ - significant difference between App+SIV-infected group and SIV-infected group; $\mathrm{f}$ - significant difference between App-infected group and SIV-infected group 
On 4 dpi, similarly to the previous rating, the concentrations of IL-8, IFN- $\gamma$, and IL-6 were significantly higher in all infected groups compared to controls $(\mathrm{P}<0.05)$ with respect to all lobes for IL-8, the middle lobe for IFN- $\gamma$, and the middle and accessory lobes for IL-6. Also, in the SIV- and App+SIV-infected groups, significantly higher concentration of IL-6 in the apical lobe was observed in comparison to controls. Moreover, significantly higher concentrations of IFN- $\gamma$ than in control pigs were detected in the SIV- and App+SIV-infected groups $(\mathrm{P}<0.05)$ with regard to the accessory and apical lobes, respectively. With respect to IL- $1 \beta$, its concentration was still significantly higher in the Appand App+SIV-infected groups compared to controls ( $\mathrm{P}<0.05)$. At this time-point, with respect to TNF- $\alpha$, a significantly higher concentration was detected only in the SIV-infected group compared to controls $(\mathrm{P}<0.05)$, whereas the concentration of IFN- $\alpha$ was still significantly higher in the SIV- and App+SIV-infected groups as compared to control group $(\mathrm{P}<0.05)$. Furthermore, similarly to the previous time-point with respect to IL-8, its concentration was significantly higher in all lobes in double-infected group compared to the single-infected groups $(\mathrm{P}<0.05)$. Similarly to $2 \mathrm{dpi}$, the concentration of IL-8 was significantly greater in the App-infected group than in the SIVinfected animals $(\mathrm{P}<0.05)$. The concentration of IFN$\alpha$ was significantly higher in the App+SIV-infected group compared to single-infected pigs $(\mathrm{P}<0.05)$. At that time-point, the differences in IFN- $\alpha$ concentration between the SIV- and App-infected groups were also statistically significant $(\mathrm{P}<0.05)$. The concentration of IL-1 $\beta$ was significantly higher in the App+SIV-infected group compared to single-infected pigs $(\mathrm{P}<0.05)$.

On day 10 , only the concentrations of IL-8, IL-1 $\beta$, and IFN- $\alpha$, in some cases, were still significantly higher compared to controls $(\mathrm{P}<0.05)$. Also, at this time-point, the concentration of IL-8 in the doubleinfected pigs was significantly higher compared to the SIV-infected and App-infected groups $(\mathrm{P}<0.05)$. Regarding IL-1 $\beta$, its concentration was significantly greater in all infected groups, compared to controls $(\mathrm{P}<0.05)$. The concentration of IFN- $\alpha$ was still significantly higher in double-infected pigs, compared to controls. Moreover, the differences in IFN- $\alpha$ concentration between double-infected and SIVinfected pigs were also still significant $(\mathrm{P}<0.05)$.

\section{Discussion}

In this study, the effect of single infection with App or SIV and dual infection with both pathogens was evaluated. There are several papers confirming the role of App and SIV as potent inducers of inflammatory mediators in the pig respiratory system $(1,9,24,25$, $29,30)$. However, to the best of the authors' knowledge, this is the first attempt to investigate the effect of the co-infection with App and SIV on the cytokine profile in the lungs.

Our data indicated that App infection alone increases the lung concentration of IL-1 $\beta$, IL-6, IL-8, and IFN- $\gamma$. Moreover, on 2 and $4 \mathrm{dpi}$, the concentration of IL-8 was significantly higher in App-infected pigs than in SIV-infected pigs. According to a previous study, expression of cytokines, such as IL-1, IL-6, and IL-8, was associated with the development of pleuropneumonia and enhancement of disease severity (1). Similarly to the current investigation, the authors of the aforementioned study evaluated the level of TNF- $\alpha$ expression and concluded that this cytokine is not actively expressed during pleuropneumoniae. Likewise, Benga et al. (5) did not detect any TNF- $\alpha$ in porcine plasma and bronchoalveolar lavage fluid (BALF) before and after infection with App. By contrast, Chen et al. (9) demonstrated the markedly increased concentration of TNF- $\alpha$, IL-1 $\beta$, and IL-8, but not IL-6, in porcine alveolar macrophage (PAM) culture supernatant stimulated with an App ApxI toxin. On the other hand, another study indicated that the experimental infection of pigs with App serotype 2 only induced detectable serum levels of IL-6 and the occurrence of IL-6 positive animals coincided with the onset of clinical signs of disease and increased body temperature (13). Furthermore, the current study indicated that pigs infected with App alone had a significantly higher concentration of lung IFN- $\gamma$ than controls. The role of IFN- $\gamma$ in the immunopathogenesis of pleuropneumoniae has not been clearly defined. In previous studies, the experimental infection of pigs with App did not induce detectable serum levels of IFN- $\gamma(2,13)$. The lack of detectable levels of IFN- $\gamma$ may be a result of the short biological half-life of this cytokine in systemic circulation (16). In another study, by contrast, a positive correlation between the plasma concentration of IFN- $\gamma$ at 4 dpi and clinical scores in pigs infected with App was observed (5). In the present study, no changes in the local lung amounts of IL-10 were observed, which is in accordance with the previous studies $(5,31)$. Contradictory findings between studies, referring to various cytokine profiles, may be due to varying study design (in vivo vs in vitro) and the components used for inoculation. The type of sample (serum and tissue homogenate) may also be important.

SIV infection alone induced inflammatory mediators with a broader range than App infection alone. Beyond IL-1 $\beta$, IL-6, IL-8, and IFN- $\gamma$, pigs infected with SIV showed increased concentrations of TNF- $\alpha$ and IFN- $\alpha$. Moreover, at 2 and $4 \mathrm{dpi}$, the concentration of IFN- $\alpha$ was significantly higher in SIVinfected pigs than in App-infected pigs. In addition, at 2 dpi, the concentration of IL- 6 was markedly higher in SIV-infected pigs, compared to App-infected pigs. A previous study in which the same virus was used for inoculation demonstrated a significant increase in IL-1 $\beta$, IL-6, IL-8, IL-10, TNF- $\alpha$, and IFN- $\gamma$ levels 
following SIV infection, and the increase in the concentrations of IL- $1 \beta$, IL- 8 , and TNF- $\alpha$ was positively correlated with lung lesions (25). The main difference between our results and the aforementioned study concerned the concentrations of IL-1 $\beta$ and IL-10. In the previous study, both cytokines were significantly elevated during the entire experiment (i.e. on 2, 7, and 10 dpi) (25). By contrast, in the present study the increments of IL-1 $\beta$ concentration were only observed at $10 \mathrm{dpi}$ and no changes in IL-10 amounts were detected at any time-point post infection. The difference in cytokine profiles may be due to the different routes of infection (intratracheal $v s$ intranasal) and type of specimen for analysis (trachea and lung tissue $v s$ only lung tissue). The early appearance of IL-1 (24-30 h post infection) was also detected by Barbé et al. (4). However, differences in the IL-1 concentration were observed in BALF, but not in the lung and serum samples. Furthermore, in pigs infected with SIV, the broader range of cytokines coincided with more severe clinical signs and lung lesions compared to App-infected animals. It may be stated that the appearance of a broader range of cytokines was responsible for excessive clinical signs and lung pathology in SIV-infected pigs compared to Appinfected pigs. However, according to Damjanovic et al. (11), TNF- $\alpha$ has an immunosuppressive role during influenza infection and the lack of this cytokine results in unregulated inflammatory responses and tissue immunopathology. Also, IL-6 plays an essential role in orchestrating anti-viral immunity, through an ability to limit inflammation, promote protective adaptive immune responses, and prevent fatal immunopathology (18). Meanwhile, a direct correlation between high morbidity and mortality of mice and high IFN $\alpha \beta$ levels, in response to influenza infection, were recorded (12). It has also been shown that in pigs infected with SIV, injection with IFN- $\alpha$ neutralising antibodies delayed the onset and peak of clinical symptoms (3).

Similarly to SIV, App+SIV infection enhanced the lung concentrations of IL-1 $\beta$, IL-6, IL-8, TNF- $\alpha$, IFN- $\alpha$, and IFN- $\gamma$ compared to controls. Moreover, coinfected pigs showed a significantly greater concentration of IL-1 $\beta$, IL- 8 , and IFN- $\alpha$ than pigs infected with either SIV or App alone. It can be assumed that a synergistic effect between App and SIV, with respect to lung inflammatory cytokines, may partially explain the more severe clinical course of disease and lung pathology than in the case of single infections. The increased severity of pneumonia, along with a significantly greater production of proinflammatory cytokines, such as IL- 8 and IFN- $\alpha$, was also observed in pigs co-infected with SIV and Bordetella bronchiseptica (17). Additionally, in that study, a positive correlation between IL- 8 and IFN- $\alpha$ transcript levels and SIV replication in the lungs was recorded. Similarly, pigs co-infected with PRRSV and bacterial LPS developed acute respiratory signs, in contrast to pigs inoculated with PRRSV or LPS only.
Moreover, the levels of TNF- $\alpha$, IL-1, and IL-6 were markedly higher in PRRSV-LPS inoculated pigs than in single-inoculated pigs, and cytokine overproduction was associated with the disease (28).

In conclusion, the obtained results indicate that the experimental infection of pigs with SIV or App alone and co-infection with both pathogens was able to induce a local lung inflammatory response. However, the local cytokine response was considerably higher in co-infected pigs, compared to single-infected pigs, suggesting that alteration in the local cytokine concentration represents an important factor through which co-infection intensifies the disease outcome. Therefore, further studies are necessary to elucidate the mechanism by which inflammatory cytokines aggravate clinical signs and lung pathology in pigs coinfected with SIV and App.

Conflict of Interests Statement: The authors declare that there is no conflict of interests regarding the publication of this article.

Financial Disclosure Statement: This work was supported by the National Science Centre (DEC2014/13/B/NZ6/02566) and by KNOW (Leading National Research Centre) Scientific Consortium "Healthy Animal - Safe Food", decision of the Ministry of Science and Higher Education no. 051/KNOW2/2015 (K/02/1.1).

Animal Rights Statement: The experiment was approved by the Local Ethics Committee at the University of Life Sciences in Lublin.

\section{References}

1. Baarsch M.J., Scamurra R.W., Burger K., Foss D.L., Maheswaran S.K., Murtaugh M.P.: Inflammatory cytokine expression in swine experimentally infected with Actinobacillus pleuropneumoniae. Infect Immun 1995, 63, 3587-3594.

2. Balaji R., Wright K.J., Turner J.L., Hill C.M., Dritz S.S., Fenwick B., Carroll J.A., Zannelli M.E., Beasang L.A., Minton J.E.: Circulating cortisol, tumor necrosis factor- $\alpha$, interleukin-1 $\beta$, and interferon- $\gamma$ in pigs infected with Actinobacillus pleuropneumoniae. J Anim Sci 2002, 80, 202-207.

3. Barbé F., Saelens X., Braeckmans D., Lefèvre F., van Reeth K.: Role of IFN-alpha during the acute stage of a swine influenza virus infection. Res Vet Sci 2010, 88, 172-178.

4. Barbé F., Atanasova K., Van Reeth K.: Cytokines and acute phase proteins associated with acute swine influenza infection in pigs. Vet J 2011, 187, 48-53.

5. Benga L., Hoeltig D., Rehm T., Rothkoetter H.J., Pabst R., Valentin-Weigand P., FUGATO-consortium IRAS. Expression levels of immune markers in Actinobacillus pleuropneumoniae infected pigs and their relation to breed and clinical symptoms. BMC Vet Res 2009, 5, 13.

6. Bossé J.T., Janson H., Sheehan B.J., Beddek A.J., Rycroft A.N., Kroll J.S., Langford P.R.: Actinobacillus pleuropneumoniae: pathobiology and pathogenesis of infection. Microb Infect 2002, 4, 225-235.

7. Bossé J.T., Li Y., Sárközi R., Gottschalk M., Angen Ø., Nedbalcova K., Rycroft A.N., Fodor L., Langford P.R.: A unique 
capsule locus in the newly designated Actinobacillus pleuropneumoniae serovar 16 and development of a diagnostic PCR. J Clin Microbiol 2017, 55, 902-907.

8. Brauer C., Hennig-Pauka I., Hoeltig D., Buettner F.F., Beyerbach M., Gasse H., Gerlach G.F., Waldmann K.H.: Experimental Actinobacillus pleuropneumoniae challenge in swine: comparison of computed tomographic and radiographic findings during disease. BMC Vet Res 2012, 8, 47.

9. Chen Z.W., Chien M.S., Chang N.Y., Chen T.H., Wu C.M., Huang C., Lee W.C., Hsuan S.L.: Mechanisms underlying Actinobacillus pleuropneumoniae exotoxin ApxI induced expression of IL-1 $\beta$, IL-8, and TNF- $\alpha$ in porcine alveolar macrophages. Vet Res 2011, 42, 25.

10. Chiers K., De Waele T., Pasmans F., Ducatelle R., Haesebrouck F.: Virulence factors of Actinobacillus pleuropneumoniae involved in colonization, persistence and induction of lesions in its porcine host. Vet Res 2010, 41, 65.

11. Damjanovic D., Divangahi M., Kugathasan K., Small C.L., Zganiacz A., Brown E.G., Hogaboam C.M., Gauldie J., Xing Z.: Negative regulation of lung inflammation and immunopathology by TNF- $\alpha$ during acute influenza infection. Am J Pathol 2011, 179, 2963-2976.

12. Davidson S., Crotta S., McCabe T.M., Wack A.: Pathogenic potential of interferon $\alpha \beta$ in acute influenza infection. Nat Commun 2014, 5, 3864.

13. Fossum C., Wattrang E., Fuxler L., Jensen K.T., Wallgren P.: Evaluation of various cytokines (IL-6, IFN-alpha, IFN-gamma, TNF-alpha) as markers for acute bacterial infection in swine-a possible role for serum interleukin-6. Vet Immunol Immunopathol 1998, 64, 161-172.

14. Gottschalk M., Lacouture S.: Actinobacillus pleuropneumoniae serotypes 3, 6, 8, and 15 isolated from diseased pigs in North America. Vet Rec 2014, 174, 452

15. Jabłoński A., Popławski R., Jędryczko R., Pomorska-Mól M., Pejsak Z: Prevalence of Actinobacillus pleuropneumoniae serovars in Poland. Abstracts Book of $24^{\text {th }}$ International Pig Veterinary Society Congress, Dublin, Ireland, 2016, p. 175.

16. Jonasch E., Haluska F.G.: Interferon in oncological practice: review of interferon biology, clinical applications, and toxicities. Oncologist 2001, 6, 34-55.

17. Kowalczyk A., Pomorska-Mól M., Kwit K., Pejsak Z., Rachubik J., Markowska-Daniel I.: Cytokine and chemokine mRNA expression profiles in BALF cells isolated from pigs single infected or co-infected with swine influenza virus and Bordetella bronchiseptica. Vet Microbiol 2014, 170, 206-112.

18. Lauder S.N., Jones E., Smart K., Bloom A., Williams A.S., Hindley J.P., Ondondo B., Taylor P.R., Clement M., Fielding C., Godkin A.J., Jones S.A., Gallimore A.M.: Interleukin-6 limits influenza-induced inflammation and protects against fatal lung pathology. Eur J Immunol 2013, 43, 2613-2625.
19. Levesque C., Provost C., Labrie J., Hernandez Reyes Y., Burciaga Nava J.A., Gagnon C.A., Jacques M.: Actinobacillus pleuropneumoniae possesses an antiviral activity against porcine reproductive and respiratory syndrome virus. PLoS One 9, 2014, e98434.

20. Loving C.L., Brockmeier S.L., Vincent A.L., Palmer M.V., Sacco R.E., Nicholson T.L.: Influenza virus coinfection with Bordetella bronchiseptica enhances bacterial colonization and host responses exacerbating pulmonary lesions. Microb Pathog 2010, 49, 237-245.

21. Madec F., Kobisch M.: Bilan lésionnel des porcs charcutiers à l'abattoir. J Rech Porc Fr 1982, 1414, 405-412.

22. Opriessnig T., Gimenez-Lirola L.G., Halbur P.G.: Polymicrobial respiratory disease in pigs. Anim Health Res Rev 2011, 12, 133-148.

23. Pomorska-Mól M., Markowska-Daniel I., Kwit K., Stępniewska K., Pejsak Z.: C-reactive protein, haptoglobin, serum amyloid A and pig major acute phase protein response in pigs simultaneously infected with H1N1 swine influenza virus and Pasteurella multocida. BMC Vet Res 2013, 9, 14.

24. Pomorska-Mól M., Kwit K., Markowska-Daniel I., Kowalski C., Pejsak Z.: Local and systemic immune response in pigs during subclinical and clinical swine influenza infection. Res Vet Sci 2014, 97, 412-421.

25. Pomorska-Mól M., Markowska-Daniel I., Kwit K., Czyżewska E., Dors A., Rachubik J., Pejsak Z.: Immune and inflammatory response in pigs during acute influenza caused by H1N1 swine influenza virus. Arch Virol 2014, 159, 2605-2614.

26. Pomorska-Mól M., Dors A., Kwit K., Kowalczyk A., Stasiak E., Pejsak Z.: Kinetics of single and dual infection of pigs with swine influenza virus and Actinobacillus pleuropneumoniae. Vet Microbiol 2017, 201, 113-120.

27. Sibila M., Aragon V., Fraile L., Segales J.: Comparison of four lung scoring systems for the assessment of the pathological outcomes derived from Actinobacillus pleuropneumoniae experimental infections. BMC Vet Res 2014, 10, 165.

28. van Gucht S., Labarque G., van Reeth K.: The combination of PRRS virus and bacterial endotoxin as a model for multifactorial respiratory disease in pigs. Vet Immunol Immunopathol 2004, $102,165-178$

29. van Reeth K., Nauwynck H.: Proinflammatory cytokines and viral respiratory disease in pigs. Vet Res 2000, 31, 187-213.

30. van Reeth K., van Gucht S., Pensaert M.: Correlations between lung proinflammatory cytokine levels, virus replication, and disease after swine influenza virus challenge of vaccinationimmune pigs. Viral Immunol 2002, 15, 583-594.

31. Wang L., Qin W., Zhang J., Bao C., Zhang H., Che Y., Sun C., Gu J., Feng X., Du C., Han W., Richard P.L., Lei L.: Adh enhances Actinobacillus pleuropneumoniae pathogenicity by binding to OR5M11 and activating p38 which induces apoptosis of PAMs and IL-8 release. Sci Rep 2016, 6, 24058. 